\title{
Bacterial Etiology of Diarrhoeal Disease in Children and Antibiogram of the Isolates
}

\author{
Anjeeta Neupane ${ }^{1^{*}}$, Pramila Parajuli ${ }^{1}$, Rama Bastola $^{1}$ and Anjan Paudel ${ }^{2}$ \\ ${ }^{1}$ Department of Microbiology, St. Xavier's College, Kathmandu, Nepal \\ ${ }^{2}$ Faculty of Pharmacy, Jamia Hamdard, New Delhi, India
}

*Corresponding author: Anjeeta Neupane, St. Xavier's College, Tribhuvan University, Kathmandu, Nepal, Tel: +9779845150048; E-mail: edu_gr8@yahoo.com

Received date: April 11, 2017; Accepted date: April 24, 2017; Published date: April 30, 2017

Copyright: ( 2017 Neupane A, et al. This is an open-access article distributed under the terms of the Creative Commons Attribution License, which permits unrestricted use, distribution, and reproduction in any medium, provided the original author and source are credited.

\begin{abstract}
Objective: The objective of this study was to determine the bacterial etiology of diarrhoeal disease in children and the antibiogram of the isolates.

Methods: The study was carried out in Bharatpur Hospital, Chitwan, Nepal form May 2014 to Oct 2014 . Stool specimens were collected from children at paedriatic ward aseptically and were processed in the Microbiology laboratory. Each sample was processed macroscopically and microbiologically. The culture of the specimen and identification of the isolates were done as per the standard guidelines and results were interpreted on the basis of different tests.
\end{abstract}

Results: Out of 202 specimens, 84 (42\%) samples showed growth and $118(58 \%)$ samples presented no any growth. Highest number of growth was observed in samples from male patients than from female patients. The predominant pathogen was Aeromonas species $33(12 \%)$, followed by NLF E. coli 19 (6.9\%), Proteus mirabilis 14 $(5.1 \%)$. Amikacin $(94 \%)$ was the most effective antibiotic and the least effective antibiotic was Amoxy-clavulanic acid $(6 \%)$. In this research, the highest multidrug resistant organism was Aeromonas species. There was significant association between diarrhoea and treatment of water $(p<0.05)$.

Conclusion: Thus, bacterial etiology of diarrhoeal disease in children and antibiogram of the isolates were determined, which was found to be an important diagnostic test for the diarroheal patient. From this study, research investigation were shown significant association between diarrhea and treatment of water, hand washing, signs and symptoms like fever, loss of appetite, abdominal cramps. Thus the study might help in the assessment of the current anti-microbial resistance patterns of bacterial isolates in diarrheal infection and helps in the formulation of the strategies to reduce the infection rate in Bharatpur Hospital.

Keywords: Diarrhoea; Enteric pathogens; Antibiotics resistant

\section{Introduction}

Diarrhoea is one of the major killer diseases of under-five children. The mortality rate for children under-five years suffering from acute Diarrhoea has fallen from 4.5 million deaths annually in 1979 to 1.6 million deaths in 2002 but continues to be highest among the children of developing countries [1]. Diarrhoea is the second most common cause of morbidity and third most common cause of mortality among children aged 12-59 months in Nepal [2]. Diarrhoea alone kills more children than AIDS, malaria, and measles combined [3]. Currently, around 1.7 billion cases of Diarrhoeal disease are reported every year and approximately 1.5 million people have annually died worldwide because of Diarrhoea [4].

Diarrhoea can also be the initial signs of non-gastrointestinal tract illness, including meningitis, bacterial pneumonia, otitis media and urinary tract infection [5]. Diarrhoea may also be caused by intestinal worms, post-infective tropical mal-absorption, lactase deficiency, antibiotic or other drug therapy which alters the normal intestinal flora, and from dietary causes including gluten intolerance [6].
Nepal, being a developing country, Diarrhoeal diseases is major problem. Both the incidence and mortality of Diarrhoeal diseases are greatest among children younger than one year of age, declining thereafter incrementally [7]. According to a recent WHO/UNICEF report, 15 countries account for almost $75 \%$ of all deaths from Diarrhoea among children below five years of age each year and more than $80 \%$ of child deaths due to Diarrhoea occur in Africa and South Asia [3].

In developing countries, approximately 2 million people, the vast majority of whom are under-five children, die from Diarrhoea each year. Nearly $90 \%$ of Diarrhoea is attributed to unsafe drinking water, inadequate sanitation and poor hygiene [8]. Diarrhoeal disease due to unsafe water and lack of sanitation is the greatest cause of morbidity and mortality in under-five children in the world, especially in poor countries $[2,8]$.

Acute infectious Diarrhoea results from various viral, bacterial, and parasitic infections and is most frequently of infectious origin. Nonetheless, in about $40 \%$ of the cases, no causative agent can be detected [9]. In addition, the recent multi country 'Global Enteric Multicenter Study' (GEMS) thoroughly investigated the causes of acute Diarrhoea in infants and young children in developing countries [10]. 
Etiological agents responsible for Diarrhoeal diseases are diverse, which includes, bacterial pathogens, parasites, viruses and fungi [11], however, the prevalence of the etiological agents varies with geographical locations, meteorological characteristics, severity of infections, and laboratory techniques employed [11]. Rotavirus is the main cause of virus-induced gastroenteritis in both developed and developing countries [12]. Escherichia coli are the most common pathogens responsible for acute Diarrhoea episodes in children; Shigella spp., Salmonella spp, Campylobacter jejuni/coli, Vibrio cholerae, Aeromonas spp, and Plesiomonas spp occur more commonly in poorer areas [9]. Assessment of stool characteristics (e.g., liquid non-bloody stools vs. dysenteric or bloody stools) is a key feature in determining potential pathogens causing an acute Diarrhoea episode [9].

The most common complication of acute Diarrhoea is dehydration [1]. Small children are least likely to tolerate large fluid shifts, consequently at least $80 \%$ of these deaths occurring in children below the age of 2 years [13].

Risk factors vary with the child's age, the pathogens involved, and the local environment. This study aimed to identify the most common bacterial pathogens, and age specific and local risk factors for Diarrhoeal disease among children aged less than twelve years admitted to Bharatpur Government Hospital, Chitwan. The infants below 6 months of age were initially protected to some extent by maternal antibodies against severe Diarrhoea, and they seem to have acquired adequate immunity between 12 and 16 months of age. The greater risk of infants and young children in the period between 6 to 12 months with declined levels of maternal antibodies have been documented [14].

Cultures take several days to provide adequate information about pathogens and their susceptibility patterns, empirical treatment must be immediately adopted in such cases. To effectively prevent Diarrhoea, it is imperative that the important risk factors associated with Diarrhoea should be identified first in communities through research.

\section{Materials and Methods}

\section{Sample collection}

A total of 202 stool samples were collected from children and infants (6 months to 12 years) suffering from Diarrhoea admitted at paediatric ward of Bharatpur Hospital. The study was carried out during May 2014 to October 2014. Stool samples collected from the patients were brought to the laboratory and processed aseptically.

\section{Sample processing}

The stool sample was processed macroscopically and microbiologically within 2 hours of its collection. On macroscopic examination, each sample was directly visualized for its appearance, colour, consistency and presence of mucus and blood. On microbiological examination, primary and secondary plating was done. On day 1 , approximately $0.5-1 \mathrm{~g}$ of sample was mixed with $2-4$ $\mathrm{ml}$ of normal saline $(0.85 \%)$ and the suspension was inoculated on MAC, HE and TCBS agar plates. For the isolation of campylobacter spp., the $0.65 \mu \mathrm{m}$ cellulose acetate membrane filter was placed centrally on BA plate and 6-8 drops separate drops were placed on the surface and was dried for 15-20 minutes. Then the inoculated plate was kept in the incubator at $37^{\circ} \mathrm{C}$ for $30 \mathrm{~min}$ to 1 hour; after then, the filter was removed.

Approximately $0.5 \mathrm{ml}$ suspension was inoculated into each tube of 0.5\% Alkaline Peptone Water (APW), Buffer Peptone Water (BPW). The inoculated MAC, HE, TCBS, $0.5 \%$ APW and BPW was incubated at $37^{\circ} \mathrm{C}$ for $16-18 \mathrm{~h}$. And, BAP in micro-aerobic condition $\left(5 \% \mathrm{O}_{2}, 10 \%\right.$ $\mathrm{CO}_{2}, 85 \% \mathrm{~N}_{2}$ ) at $37^{\circ} \mathrm{C}$ for up to $72 \mathrm{~h}$.

On day 2, a drop of $0.5 \%$ APW (from day 1) was inoculated on TCBS agar. In the same way a drop of BPW broth was inoculated on $\mathrm{MAC}$ and $\mathrm{HE}$ agar, and streaking was done and were then incubated in the same condition as done on first day.

The primary cultured plates of MAC, HE, TCBS were examined macroscopically and the colony morphology was recorded which resemble that of Salmonella, Shigella, Vibrio, E. coli, Aeromonas, Plesiomonas. The colony present on these plates was stained. The isolated colonies (non-lactose fermenting and lactose fermenting) on the MAC, HE, TCBS agar was picked and inoculated onto TSI slant. The inoculated TSI media were incubated at $37^{\circ} \mathrm{C}$ for $16-18 \mathrm{~h}$.

On day 3 , the reaction on TSIA (NLF colonies picked on day 2) was noted to find whether the reaction was (acid butt and alkali slant on TSIA media) with or without $\mathrm{H}_{2} \mathrm{~S}$ and gas. The reaction of TSIA (LF colonies picked on day 2) was noted, if the reaction was A/A (acid reaction both of butt and slant), without $\mathrm{H}_{2} \mathrm{~S}$, with gas or no gas, The oxidase test was performed and then the colony was inoculated to a set of biochemical media like SIM, Methyl Red, Voges-Proskauer, Simmon's Citrate (IMViC), Lysine Deoxycolase (LD), Urease test, Oxidation-Fermentation test (OF). The TSIA whose reaction is $\mathrm{K} / \mathrm{K}$ (alkali butt and alkali slant) was discarded.

The sub-cultured plates of MAC, HE, TCBS (sub-cultured on day 2) were noted, further steps were performed in the manner done for primary plating. The inoculated biochemical tubes were incubated at $37^{\circ} \mathrm{C}$, overnight. BAP media (from day 1 ), were examined for Campylobacter spp. that produce gray, flat, irregular, spreading colonies, particularly on freshly prepared media. Haemolysis on blood agar was not observed.

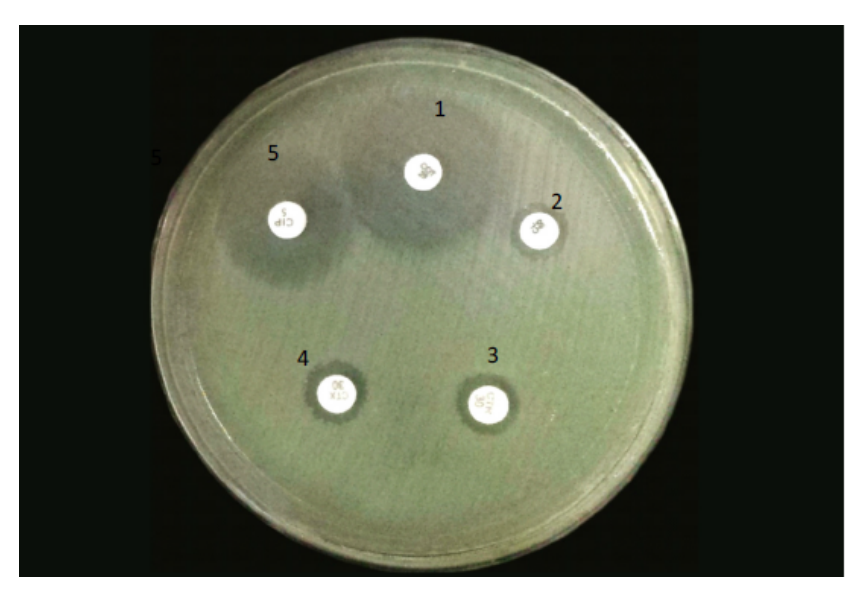

Figure 1: Antibiotic susceptibility testing of NLF E. coli in MHA plate. Sensitive: Cotrimoxazole (1), Ciprofloxacin (5). Resistant: Colistin (2), Ceftriaxone (3), Cefotaxime (4). 
On day 4, the biochemical reactions of NLF and LF isolates performed on the third day were noted and then Antibiotic susceptibility test (Figures 1 and 2) was performed.

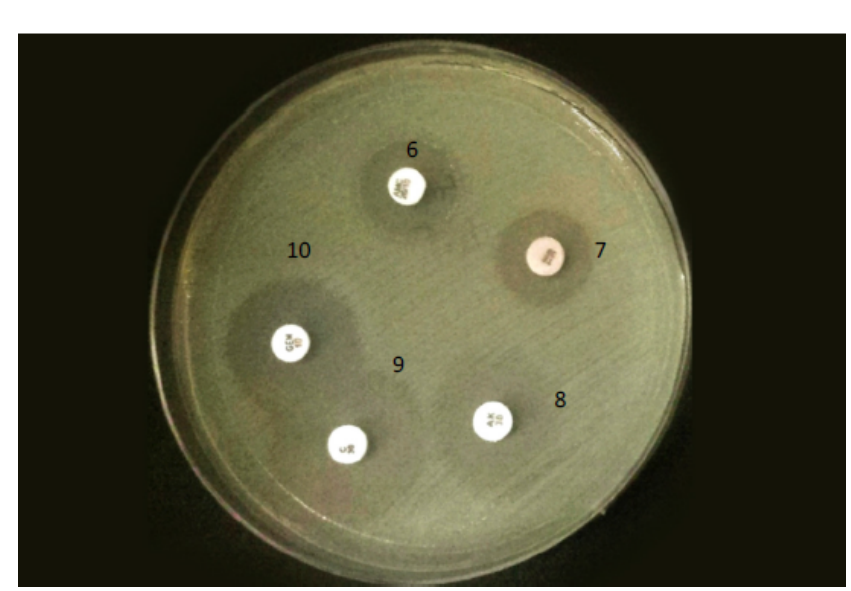

Figure 2: Antibiotic susceptibility testing of NLF E. coli in MHA plate. Sensitive: Amikacin (8), Chloramphenical (9), Gentamicin (10). Resistant: Amoxyclavulinic acid (6), Tetracycline (7).

\section{Antibiotic susceptibility testing}

Antibiotic treatment of common bacterial infections plays a crucial role in reducing morbidity and mortality of diseases; however, overuse and misuse of antibiotics in the treatment of Diarrhoea could lead to increased antibiotic resistance. Antibiotic susceptibility testing was performed using the standard disc diffusion method recommended by the Clinical and Laboratory Standard Institute for the following antibiotics: Amikacin, Amoxy-clavulanic acid, Gentamicin, Ciprofloxacin, Chloramphenical, Cefotaxime, Ceftriaxone, Colistin, Tetracycline and Cotrimoxazole.

The MHA plate with $4 \mathrm{~mm}$ depth of medium was taken. The inoculum was prepared and was standardized to match the 0.5 MacFarland turbidity standards. A sterile cotton swab was dipped into the suspension and uniform swabbing was done. With the help of flamed forcep, discs were carefully placed on the inoculated plate and pressed gently into agar. The plates were allowed to stand at room temperature for 30 minutes. The plates were incubated at $37^{\circ} \mathrm{C}$ for 24 $\mathrm{h}$, and the diameters of zone of inhibition were compared with those of the reference isolate (E. coli ATCC 25922). Susceptibility results were reported as sensitive, intermediate and resistant.

\section{Quality control}

A standard bacteriological procedure was followed to keep the quality of all laboratory tests. The quality control was performed in every required step. The samples were collected using sterile leak proof container aseptically in order to avoid contamination. The sterility of each batch of the test medium was confirmed by incubating uninoculated plates and tubes overnight at $37^{\circ} \mathrm{C}$ and was not used if those plates and tubes showed the evidence of bacterial growth and other visual reactions after incubation. The positive and negative controls were incubated along with test for comparing the results. American Type Culture Collection (ATCC) strain (E. coli ATCC 25922) was used as control strains for the culture and sensitivity testing. Similarly, indicator media with correct $\mathrm{pH}$ antibiotics discs having correct amount as indicated was used.

\section{Data analysis}

Data was collected, summarized, tabulated and analyzed. Analysis of the data was carried out using software, namely; Statistical Package for Social Sciences (SPSS) version 20. The results were presented through tables, pie charts and bar graph. Comparisons of variables were tested by Chi-square $\left(\chi^{2}\right)$ test and $p$ value less than 0.05 were considered significant.

\section{Results}

In this study, a total of 202 stool samples were collected from children and infants suffering from Diarrhoea with different signs and symptoms admitted at paediatric wards of Bharatpur Hospital.

\section{Pattern of growth in stool samples}

Out of 202 samples the growth observed in 84 (41.6\%) samples and no growth observed in 118 (58.4\%) samples (Figure 3).

\section{\begin{tabular}{|l|l|}
\hline \multicolumn{2}{|c|}{ Growth Pattern } \\
$58.4 \% \quad \square$ Growth \\
\\
Figure 3: Pattern of growth in stool samples.
\end{tabular}}

\section{Sex wise distribution of patients}

Out of 202 samples, 117 (57.9\%) were specimens from male patients and $85(42.1 \%)$ were from female patients. Among the 84 culture positive samples, $46(39.3 \%)$ were specimens from male patients and 38 (43.9\%) were from females (Table 1). The growth was found to be higher in specimens from male patients than in female patients but higher incidence was found in samples of female patients. The prevalence of Diarrhoeal disease was not significantly affected by gender $(\mathrm{p}=0.443)$.

\begin{tabular}{|l|l|l|l|l|}
\hline Gender & Growth & No Growth & Total & P-value \\
\hline Male & 46 & 71 & 117 & \\
\hline Female & 38 & 47 & 85 & 0.443 \\
\hline Total & $\mathbf{8 4}$ & $\mathbf{1 1 8}$ & $\mathbf{2 0 2}$ & \\
\hline
\end{tabular}

Table 1: Sex wise distribution of patients. 
Citation: $\quad$ Neupane A, Parajuli P, Bastola R, Paudel A (2017) Bacterial Etiology of Diarrhoeal Disease in Children and Antibiogram of the Isolates.

Page 4 of 8

\section{Age wise distribution of patients}

The maximum number of Diarrhoeal samples (93) was collected from the age group 12-48 months in which growth was obtained in 48 (51.6\%) samples (Table 2). The highest incidence of Diarrhoeal disease was recorded in the age group 108-144 months (71.4\%) while lowest incidence was in the age group 60-96 months (5-8 years), being $33.3 \%$. A significant association was found between the age and prevalence of Diarrhoeal diseases $(\mathrm{p}<0.05)$.

\begin{tabular}{|c|c|c|c|c|}
\hline \multirow{2}{*}{ Age (months) } & Growth & No Growth & Total & P-value \\
\hline & No. & No. & No. & \multirow{6}{*}{0.008} \\
\hline$<12$ & 24 & 57 & 81 & \\
\hline $12-48$ & 48 & 45 & 93 & \\
\hline $60-96$ & 7 & 14 & 21 & \\
\hline $108-144$ & 5 & 2 & 7 & \\
\hline Total & 84 & 118 & 202 & \\
\hline
\end{tabular}

Table 2: Age wise distribution of patients.

\section{Bacterial isolates in Diarrhoeal disease}

\section{Distribution of enteric organisms in Diarrhoeal stool sample}

Out of 84 culture positive samples collected from patients suffering from Diarrhoea, Aeromonas species $(33,16.3 \%)$ as shown in Figure 4 was the most predominant species followed by $N L F E$. coli $(19,9.4 \%)$, Proteus mirabilis (14, 6.9\%), Other Salmonella species (6, 3.0\%), Edwardsiella species (4, 2.0\%), Shigella species (2, 1.0\%), Proteus vulgaris (2, 0.7), Salmonella Typhi (1, 0.5\%), Salmonella Paratyphi (1, $0.5 \%)$, Citrobacter species (1, 0.5\%), Plesiomonas shigelloides ( 1 , $0.5 \%)$.

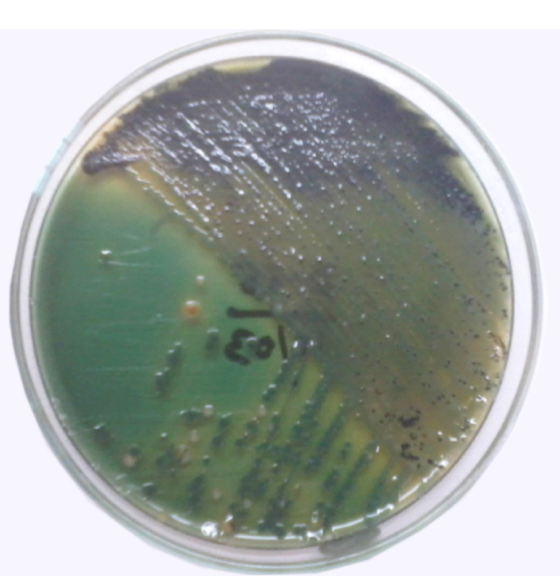

Figure 4: Hekteon enteric agar plate showing greenish 3-4 mm opaque, moist, round, smooth and convex colony of Aeromonas species (Sample no. 103/202).

\section{Correlation between Diarrhoea and source of drinking water}

The maximum number of enteric pathogens were isolated in patients using Municipal water supply for drinking purposes 32 (36\%), but the highest incidence of growth was in open well users $3(75 \%)$.
There was no significant association between Diarrhoea and sources of drinking water (Table 3).

\begin{tabular}{|l|l|}
\hline Organisms & No of isolates \\
\hline Aeromonas species & 33 \\
\hline Citrobacter species & 1 \\
\hline Edwardsiella species & 4 \\
\hline NLF E. coli & 19 \\
\hline Plesiomonasshigelloides & 1 \\
\hline Proteus mirabilis & 14 \\
\hline Proteus vulgaris & 2 \\
\hline Salmonella Typhi & 1 \\
\hline Salmonella Paratyphi & 1 \\
\hline Other Salmonella species & 6 \\
\hline Shigella species & 2 \\
\hline Total & $\mathbf{8 4}$ \\
\hline
\end{tabular}

Table 3: Distribution of enteric organisms isolated in Diarrhoeal stool samples.

Most of patients used treated drinking water for drinking purposes and the number of growth of pathogens were higher in untreated water $61(63.5 \%)$ than boiling water $20(25.6 \%)$ and filtered water $3(10.7 \%)$ users (Table 4). There was significant association between Diarrhoea and treatment process $(\mathrm{p}<0.05)$.

\begin{tabular}{|c|c|c|c|c|}
\hline Drinking water & Growth & No Growth & Total & P-value \\
\hline \multicolumn{4}{|l|}{ Sources } & \multirow{6}{*}{0.059} \\
\hline Tube well & 24 & 17 & 41 & \\
\hline Public city supply & 25 & 42 & 67 & \\
\hline Government tap water & 32 & 57 & 89 & \\
\hline Open well & 3 & 1 & 4 & \\
\hline Dhungedhara & - & 1 & 1 & \\
\hline \multicolumn{4}{|l|}{ Treatment } & \multirow{4}{*}{0} \\
\hline Boiling & 20 & 58 & 78 & \\
\hline Filtration & 3 & 25 & 28 & \\
\hline No treatment & 61 & 35 & 96 & \\
\hline
\end{tabular}

Table 4: Source of drinking water.

\section{Correlation between Diarrhoea and sanitation practices}

The highest incidence of enteric pathogens was isolated in patients using open surrounding $4(66.7 \%)$ for toilet practice followed by baby pan $24(50 \%)$, cloth diaper $38(40 \%)$, toilet users $15(34.9 \%)$, and disposable diapers users 3 (30\%) (Table 5). 


\section{Antimicrobial susceptibility pattern}

\section{Antimicrobial susceptibility pattern of total isolates}

The antibiotic discs used were Amikacin, Ciprofloxacin, Ceftriaxone, Tetracycline, Cotrimoxazole, etc. (Table 6). Among these, Amikacin (94\%) was the most effective antibiotic followed by Chloramphenical (76.2\%), Gentamicin (75\%), Cefotaxime (70.2\%) and the least effective antibiotic was Amoxyclavuliniic acid (6\%).

\begin{tabular}{|c|c|c|c|c|}
\hline Sanitation practice & Growth & No Growth & Total & $P$ value \\
\hline \multicolumn{4}{|l|}{ Toilet practice } & 0.355 \\
\hline Cloth diaper & 38 & 57 & 95 & \\
\hline Disposable diaper & 3 & 7 & 10 & \\
\hline Uses toilet & 15 & 28 & 43 & \\
\hline Baby pan & 24 & 24 & 48 & \\
\hline Surrounding & 4 & 2 & 6 & \\
\hline
\end{tabular}

Table 5: Sanitation practices in the house.

\begin{tabular}{|l|l|l|l|}
\hline Antibiotics & Sensitive & Intermediate & Resistant \\
\hline Amikacin & 79 & - & 5 \\
\hline Cotrimoxazole & 41 & 5 & 38 \\
\hline Ceftriaxone & 54 & 16 & 14 \\
\hline Ciprofloxacin & 52 & 7 & 25 \\
\hline Cefotaxime & 59 & 8 & 17 \\
\hline Chloram-phenical & 64 & - & 20 \\
\hline Colistin & 30 & - & 54 \\
\hline Tetracycline & 34 & 2 & 48 \\
\hline Gentamycin & 63 & 5 & 16 \\
\hline Amoxyclav & 5 & 9 & 70 \\
\hline
\end{tabular}

Table 6: Antimicrobial susceptibility pattern of total isolates.

\section{Multi-Drug Resistant organisms}

The highest no. of MDR isolates was obtained in Aeromonas spp. 26 (78.8\%) followed by Salmonella spp. 6 (75\%), NLF E. coli 14 (73.7\%), Proteus mirabilis 10 (71.4\%), Shigella spp. 1 (50\%) (Figure 5).

\section{Discussion}

The present study of Diarrhoeal diseases was carried out on infants and children who were suffering from Diarrhoea at paediatric ward of Bharatpur Hospital, Chitwan. A total of 202 stool samples were collected and processed macroscopically and microbiologically. They were cultured in appropriate culture media, identification was made and antimicrobial susceptibility testing was carried out for appropriate selection of antibiotic.

In this study, out of 202 stool samples, $84(41.6 \%)$ were culture positive and 118 (58.4\%) were culture negative. Culture negative were higher than culture positive which may be because of antibiotic administration before admission to hospital and commensal organisms were avoided in the study.

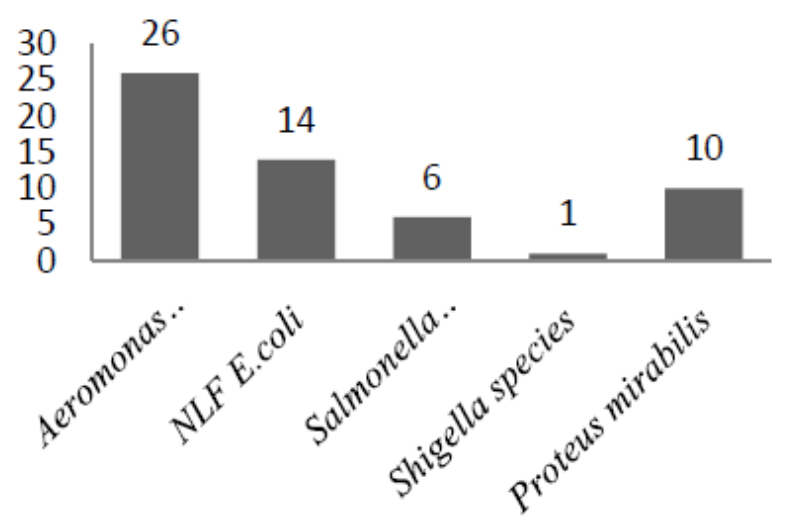

Figure 5: Bar graph showing number of MDR organisms.

In the study carried out by Okon et al. [15] in which out of 144 specimens, enteropathogens were found in 89 (61.8\%) and 55 (38.2\%) cases yielded negative results. Of the 89 enteropathogens detected 48 (53.9\%) were bacterial pathogens which were different from the result of current study. In the study carried out by Sawsan et al. [16], five hundred samples of stool were collected from patients with Diarrhoea (infants and children under ten years of age) admitted to the Paediatric and Maternity Hospital in Erbil City. No infectious agents were found in $75(15 \%)$ of the samples which is different from the result of present study. In the study reported by Nair et al. [17] in Kolkata, India where $27.9 \%$ of the Diarrhoea patients had no potential pathogen which is different from the result of present study.

In this study, among the 84 culture positive cases, 46 (39.3\%) were specimens from males patients and 38 (44.7\%) were from females. The growth was found to be higher in specimens obtained from male patients than in female patients but the higher incidence of growth was obtained in female patients. This may be because sample number from male patients were higher in number than female patients. The maximum number of culture positive samples (93) were observed in children within the age group 12-48 months, out of which 48 (51.6\%) were males and $45(48.4 \%)$ were females. This may be because of this age-group has high possibility of contact with contaminated food and drinks, and soil due to their daily outdoors activities.

Similar study was carried out by Okon et al. [15], over one year period from January to December, 2010, in which out of 144 samples, the sex distribution was $80(55.5 \%)$ males and 64 (44.4\%) female respectively. In this high prevalence of enteropathogens were observed higher among males 80 (55.5\%) and children within the age-group $12-48$ months 71 (49.3\%). Similar findings was obtained in the study of Ansari et al. [18] in Kathmandu Nepal, boys had higher Diarrhoeal cases (64.2\%) than girls (35.8\%). Kumar et al. [19] conducted the study in India, in which out of 200 cases male comprised 59.5\% and female $40.5 \%$. So male: female ratio was $1.5: 1$. Similarly, in a study carried out by Kansakar et al. [20], out of 877 stool specimens, 148 (16.8\%) were culture positive for the bacterial entero-pathogens investigated in the study. Of the 148 culture positive stool specimens, 87 were from males and 61 were from females. 
In a study by Gebrekidan et al. [21] stool specimens were collected from 216 patients Out of which, 109 (50.5\%) were male and 107 (49.5 \%) were female. In the study carried out by Cajetan et al. [22] in Abuja, Nigeria in which 184 (45.5\%) were male and 220 (54.5\%) were female which is different from the result of the present study.

In the present study, the maximum number of Diarrhoeal samples (93) was collected from the age group 12-48 months in which growth was obtained in 48 (51.6\%) Diarrhoeal samples. The highest incidence of Diarrhoeal disease was recorded in the age group 108-144 months (71.4\%) while lowest incidence was in the age group 60-96 months (5-8 years), being $33.3 \%$ which increases with increase in age 9-12 years of the patient to $71.4 \%$. A significant association was found between the age and prevalence of Diarrhoeal diseases $(\mathrm{p}<0.05)$.

In a study carried out by Okon et al. [15] in which the age-group distribution of the patients was as follows: $12-48$ months, $71(49.3 \%)$; 60-96 months, 41 (28.5\%); 108-144 month, 22 (15.3\%) and <12 month, $10(6.9 \%)$ which is comparable with the current study. Shirley Karambu et al. [23], in a study carried out in Igembe District Hospital in Meru found that the prevalence rate was lowest in over 4 year-old implying that children aged between 12 months and 48 months were more susceptible to Diarrhoeal than those $>4$ years of age which was in accordance with the present study. A study carried out at Erbil city, by Sawsan et al. [16] found that Diarrhoea is common in the age group less than 3 years. The highest percentage of infection was found in the first, second, and third age groups by different causes which is comparable with the present study.

In the study carried by Sherchand et al. [14] at Kanti Children's Hospital between May to October 2007, where they reported the age group 6-8 years was found highly infected (19.4\%) which is different from the present study.

In this study, out of 84 culture positive samples, collected from patients suffering from Diarrhoea, the most commonly isolated pathogens were Aeromonas spp. (33, 16.3\%), NLF E.coli (19, 9.4\%), Proteus mirabilis (14, 6.9\%), Other Salmonella spp. (6, 3.0\%), Edwardsiella spp. (4, 2.0\%), Shigella spp. (2, 1.0\%), Proteus vulgaris (2, 0.7\%), Salmonella Typhi (1, 0.5\%), Salmonella Paratyphi (1, 0.5\%), Citrobacter spp. (1, 0.5\%), Plesiomonasshigelloides (1, 0.5\%).

Taneja et al. [24] conducted a study in PGIMER, Chandigarh from January 2000 to September 2002 in which total of 1802 faecal samples from the same number of patients suffering from Diarrhoea submitted to the Department of Medical Microbiology, in which they reported Aeromonas spp. (14), Salmonella Typhi (2) and Salmonella Paratyphi (2).

In the study carried out by Sherchand et al. [14]; the higher incidence of Shigella spp. (36.8\%) and Salmonella spp(14.03\%) which was different from the present study. Similarly, in a study carried out by Okon et al. [15], Salmonella spp. accounts for 1 (0.4\%) which was in accordance with our study. In a study by Kumar et al. [19] Shigella (7.5\%), Salmonella (7.5\%), Proteus (5.5\%), were isolated which was comparable with this study. The variation in different series might be due to the variation in place, time and season pattern of feeding and socio-economic status of the cases.

In the present study, maximum number of enteric pathogens were isolated in patients using Government water for drinking purposes 32 (36\%), but the highest incidence of growth was in open well users 3 (75\%). There was no significant association between Diarrhoea and sources of drinking water.
In a similar study by Siziya et al. [25] has compared to children who belonged to households with unprotected well or river as the main source of water, children who belonged to households with piped water were $32 \%$ more likely to have Diarrhoea while those who belonged to households with protected well were $26 \%$ less likely to have Diarrhoea. Garrett et al. [26] in Kenya showed that Diarrhoea risk was higher among shallow well user which was in accordance with the present study. Different findings were observed by Abdelateef [27], in which tap water in Gaza strip may be contaminated because the per cent of cases drinking tap water are more frequent than those in controls (11.4\% in cases, $9.8 \%$ in controls). In addition, there is statistically significant correlation between using domestic wells water for drinking purposes and Diarrhoea.

In this study, the patients used treated drinking water for drinking purposes were higher and the number of growth of pathogens were higher in untreated water 61 (63.5\%) than boiling water $20(25.6 \%)$ and filtered water 3 (10.7) users. There was significant association between Diarrhoea and treatment process $(\mathrm{p}<0.05)$.

In the study carried out by Karambu et al. [23], Kenya treatment of water before drinking was done by about half of the study participants $(50.9 \% \mathrm{n}=157)$ with majority of participants identifying with boiling method (79.6\%) however none of the method was found to have any association with Diarrhoea which is different from the present study.

In the present study, most of the participants wash their hands always, some of them wash their hands frequently and some wash rarely. The incidence of enteric pathogens was higher in children who wash their hands frequently (53) followed by those who wash their hands rarely (4). A significant association was found between incidence of enteric pathogen and frequency of hand washing with soap by child. Although most of the patient's guardian washed their hands always and the incidence of enteric pathogens was higher in mother who washed their hands frequently 21 (91.3\%). A significant association was found between incidence of enteric pathogen and frequency of hand washing with soap by mother.

Similar results were given by Gebru et al. [28], in the comparative cross-sectional study at southwest Ethiopia, where a remarkable difference of childhood Diarrhoea were observed among children whose mothers not practiced hand washing at critical time with soap were more likely to develop Diarrhoea when compared to children whose mothers were practiced hand washing at critical time with soap. Similarly in a study by Karambu et al. [23], care taker not washing hands after changing napkin can expose the child to Diarrhoea illness more than one and half times as compared to those washing hands after changing the napkins.

The overuse and misuse of antibiotics in the treatment of Diarrhoea could lead to an increase of antibiotic resistance [29,30]. Limited laboratory diagnosis in developing countries imposes clinicians to syndromaic diagnosis and empirical prescription of broad spectrum antibiotics that led drug resistant bacterial strains to emerge [1].

Antimicrobial susceptibility testing is important for the correct prescription of antibiotics for the treatment of patients. Antibiotic sensitivity testing is an in vitro method for estimating the activity of drugs which will assist clinician in selecting an antimicrobial agent effective in inhibiting the growth of an infecting microorganism in vivo. The aim of antimicrobial therapy is to choose a drug which is selectively active against the most likely pathogens and least likely to cause adverse effects or promote resistance. Hence it is necessary to 
determine the antibiotic susceptibility of organisms isolated from infected patients.

In this study, the antibiotic discs used were Amikacin, Ciprofloxacin, Ceftriaxone, Tetracycline, Cotrimoxazole, etc. Among these, Amikacin (94\%) was the most effective antibiotic followed by Chloramphenical (76.2\%), Gentamicin (75\%), Cefotaxime (70.2\%) and the least effective antibiotic was Amoxyclavuliniic acid (6\%).

In a study carried out by Samie et al. [31] observed Ceftriaxone (91\%) and Ciprofloxacin (89\%) showed stronger activities and Gentamycin (81\%) which was comparable with the study. Abdelateef [27], reported that the antimicrobial profile of the isolated enteropathogenic bacteria showed that antibiotics resistance to Tetracycline $(66.6 \%),(16.6 \%)$ to Chloramphinicol, (16.6\%) to Cefatoxime, $(8.3 \%)$ to Ceftriaxone, $(8.3 \%)$ to Ciprofloxacin. All isolates were sensitive to Gentamicin, and Amikacin which were in accordance with the study.

Different findings were observed by Karambu et al. [23] in which was resistance in Cotrimoxazole and Tetracycline (96\%, 81\%).

In this study all isolates of other Salmonella species were $100 \%$ sensitive to Gentamycin followed by Amikacin, Ceftriaxone, Cefotaxime, Chloramphenical, and Tetracycline (66.7\%, each). Highest resistance, with $66.7 \%$ was seen with colistin followed by Cotrimoxazole showing resistance with 50\%. All isolates were resistance to Amoxyclavulinic acid.

Ansari et al. [18] reported that, among the Salmonella species, Chloramphenicol and Tetracycline showed efficacy in $9(90.0 \%)$ isolates in vitro followed by Amikacin in 8 (80.0\%) isolates. Similarly in the study by Urio et al. [32] the Salmonella species were susceptible to Chloramphenicol, Colistin-sulphate, Gentamicin.

In the present study, the two shigella species were isolated and were $100 \%$ sensitive to Ceftriaxone, Chloramphenical, and Gentamycin and $50 \%$ resistance to Amikacin, Cotrimoxazole, Ciprofloxcin, Cefotaxime, Colistin, Tetracyline. The isolates were $100 \%$ resistance to Amoxyclavulinic acid.

Similar findings were observed by Ansari et al. [18] in which among the total Shigella species, $22(91.7 \%)$ isolates were susceptible to Gentamycin followed by $21(87.5 \%)$ isolates susceptible to Amikacin whereas $12(50.0 \%)$ isolates resistant to Cotrimoxazole. In another study by Kansakar et al. [20], all the Shigella isolates were susceptible to Ceftriaxone and Ciprofloxacin resistance was seen among $20 \%$ and $24 \%$ of the isolates. All nine Salmonella isolates were susceptible to Ciprofloxacin and Ceftriaxone. Out of five Salmonella typhi identified, three were MDR showing simultaneous resistance to Chloramphenicol, Cotrimoxazole. Similarly, in a study by Urio et al. [32] in Gaborone, Botswana, Shigella species were susceptible to Chloramphenicol. Seven isolates of Shigella (12.7\%) were resistant to Ciprofloxacin, (18.5\%) of non-typhoidal isolates were resistant to ciprofloxacin. Different findings were observed by Gebrekidan et al. [21] in which low resistance was observed to Gentamicin (13.3\%) and Amoxicillin clavulanic acid (33.3\%).

The highest no. of MDR isolates was obtained in Aeromonas spp. 26 (78.8\%) followed by Salmonella spp. 6 (75\%), NLF E. coli 14 (73.7\%), Proteus mirabilis 10 (71.4\%), Shigella spp. 1 (50\%).

Similar findings were observed by Ansari S, et al. [18] in which MDR was highest 7 (70.0\%) in Salmonella spp. followed by 14 (58.3\%) in Shigella spp.

\section{Acknowledgement}

I would like to express my special appreciation and thanks to Department of Microbiology, St. Xavier's College.

\section{References}

1. Okeke IN, Aboderin OA, Byarugaba DK, Ojo KK, Opintan JA (2007) Growing problem of multidrug-resistant enteric pathogens in Africa. Emerg Infect Dis 13: 1640-1646.

2. Tumwine JK, Thompson J, KatuaKatua M, Mujwajuzi M, Johnstone N, et al. (2002) Diarrhoea and effects of different water sources, sanitation and hygiene behaviour in East Africa. Trop Med Int Health 7: 750-756.

3. WHO (2009) Diarrhoea: why children are still dying and what can be done. Geneva: UNICEF/WHO.

4. World Health Organization Diarrhoeal disease (2013).

5. Centers for Disease Control and Prevention (2004) Managing acute gastroenteritis among children: oral rehydration, maintenance, and nutritional therapy. Pediatrics 114: 507-507.

6. Cheesbrough M (2006) District Laboratory Practice in Tropical Countries, Part I, 2nd edition, Cambridge University Press, New York.

7. Farthing M, Lindberg G, Dite P, Khalif I, Salazar-Lindo E, et al. (2008) World Gastroenterology Organisation practice guideline: acute Diarrhoea. Milwaukee, Wisconsin, USA: World Gastroenterology Organisation 7-12.

8. Ahs JW, Tao W, Löfgren J, Forsberg BC (2010) Diarrhoeal diseases in lowand middle-income countries: incidence, prevention and management. Opn Infect Dis J 4: 113-124.

9. O'Ryan M, Prado V, Pickering LK (2005) A millennium update on pediatric Diarrhoeal illness in the developing world. Semin Pediatr Infect Dis 16: 125-136.

10. Kotloff KL, Blackwelder WC, Nasrin D, Nataro JP, Farag TH, et al. (2012) The Global Enteric Multicenter Study (GEMS) of Diarrhoeal disease in infants and young children in developing countries: epidemiologic and clinical methods of the case/control study. Clin Infect Dis 55: S232-S245.

11. Reither K, Ignatius R, Weitzel T, Seidu-Korkor A, Anyidoho L, et al. (2007) Acute childhood Diarrhoea in northern Ghana: epidemiological, clinical and microbiological characteristics. BMC Infect Dis 7: 104.

12. Erdem B, KARİTAŞ E, Cil E, IŞIK K (2011) Biochemical identification and numerical taxonomy of Aeromonas spp. isolated from food samples in Turkey. Turk J Biol 35: 463-472.

13. Seear M (2000) A Manual of Tropical Pediatrics, Second Edition. pp 126-136.

14. Sherchand JB, Yokoo M, Sherchand O, Pant AR, Nakogomi O (2009) Burden of enteropathogens associated Diarrhoeal diseases in children hospital, Nepal. Scientific World 7: 71-75.

15. Okon KO, Nguru MG, Bularafa MY, Mohammed HU, Baba ZA, et al. (2014) Enteropathogens Associated with Childhood Diarrhoeal Cases seen at a Tertiary Hospital in Nguru, Yobe State of Nigeria. Brit Microbiol Res J 4: 583.

16. Al-Sorchee SMA, Rabat AA, Juma IM (2013) Microbial Causatives of Diarrhoea in Children in Erbil City. J Al-Nahrain Uni 16: 19-29.

17. Nair GB, Ramamurthy T, Bhattacharya MK, Krishnan T, Ganguly S, et al. (2010) Emerging trends in the etiology of enteric pathogens as evidenced from an active surveillance of hospitalized Diarrhoeal patients in Kolkata, India. Gut Pathog 2: 4.

18. Ansari S, Sherchand JB, Parajuli K, Mishra SK, Dahal RK, et al. (2013) Bacterial etiology of acute Diarrhoea in children under five years of age. J Nepal Health Res Counc 10: 218-223.

19. Kumar M, Ramkrishana M, Koram LK (2014) Study of Various Causative Factors of Diarrhoeal Diseases with Special Reference to Bacteriological Profile among 0 to 5 Years Age Group. Int J Sci Stud 2: 47-52.

20. Kansakar P, Baral P, Malla S, Ghimire GR (2011) Antimicrobial susceptibilities of enteric bacterial pathogens isolated in Kathmandu, Nepal, during 2002-2004. J Infect Dev Ctries 5: 163-168. 
Citation: $\quad$ Neupane A, Parajuli P, Bastola R, Paudel A (2017) Bacterial Etiology of Diarrhoeal Disease in Children and Antibiogram of the Isolates.

21. Gebrekidan A, Dejene TA, Kahsay G, Wasihun AG (2015) Prevalence and antimicrobial susceptibility patterns of Shigella among acute Diarrhoeal outpatients in Mekelle hospital, Northern Ethiopia. BMC Res Notes 8: 611.

22. Cajetan ICI, Nnennaya IR, Casmir AA, Florence IN (2010) Enteric bacteria pathogens associated with Diarrhoea of children in the federal capital territory Abuja, Nigeria. N Y Sci J 3.

23. Karambu S, Matiru V, Kiptoo M, Oundo J (2014) Characterization and factors associated with Diarrhoeal diseases caused by enteric bacterial pathogens among children aged five years and below attending Igembe District Hospital, Kenya. Pan Afr Med J 16: 37.

24. Taneja N, Mohan B, Khurana S, Sharma M (2004) Antimicrobial resistance in selected bacterial enteropathogens in north India. Indian J Med Res 120: 39-43.

25. Siziya S, Muula AS, Rudatsikira E (2009) Diarrhoea and acute respiratory infections prevalence and risk factors among under-five children in Iraq in 2000. Ital J Pediatr 35: 8.

26. Garrett V, Ogutu P, Mabonga P, Ombeki S, Mwaki A, et al. (2008) Diarrhoea prevention in a high-risk rural Kenyan population through point-of-use chlorination, safe water storage, sanitation, and rainwater harvesting. Epidemiol Infect 136: 1463-1471
27. Abdelateef N (2011) Antimicrobial Resistance for Enteric Pathogens Isolated from Acute Gastroenteritis Patients in Gaza strip, Palestine (Doctoral dissertation, The Islamic University-Gaza).

28. Gebru T, Taha M, Kassahun W (2014) Risk factors of Diarrhoeal disease in under-five children among health extension model and non-model families in Sheko district rural community, Southwest Ethiopia: Comparative cross sectional study. BMC Public Health 14: 395.

29. Ecker L, Olarte L, Vilchez G, Ochoa TJ, Amemiya I, et al. (2011) Physician's responsibility for antibiotic use in infants from periurban Lima, Peru. Rev Panam Salud Publica 30: 574-579.

30. Reda AA, Seyoum B, Yimam J, Andualem G, Fiseha S, et al. (2011) Antibiotic susceptibility patterns of Salmonella and Shigella isolates in Harar, Eastern Ethiopia. J Infect Dis Immun 3: 134-139.

31. Samie A, Guerrant RL, Barrett L, Bessong PO, Igumbor EO, et al. (2009) Prevalence of intestinal parasitic and bacterial pathogens in Diarrhoeal and non-Diarrhoeal human stools from Vhembe district, South Africa. J Health Popul Nutr 27: 739-745.

32. Urio EM, Collison EK, Gashe BA, Sebunya TK, Mpuchane S (2001) Shigella and Salmonella strains isolated from children under 5 years in Gaborone, Botswana, and their antibiotic susceptibility patterns. Trop Med Int Health 6: 55-59. 